\title{
Mortalidad neonatal y factores asociados, Macuspana, Tabasco, México
}

\author{
Neonatal mortality and associated factors in Macuspana, \\ Tabasco, Mexico
}

\author{
Carlos Mario de la Cruz Gallardo' ; Victor Hugo Robles Calvillo"; J osé Alejo \\ Hernández Blé!l' \\ 'Especialita en Salud Pública. Secretaría de Salud del Gobierno del Estado de \\ Tabasco. México. \\ "Maestro en Ciencias en Epidemiología. Secretaría de Salud del Gobierno del Estado \\ de Tabasco. México. \\ "IIMaestro en Ciencias en Salud Pública. Epidemiólogo de la Jurisdicción Sanitaria de \\ Macuspana. Secretaría de Salud del Gobierno del Estado de Tabasco. México.
}

\section{RESUMEN}

I ntroducción La mortalidad neonatal constituye un problema de salud mundial y a la vez es un indicador de calidad de la atención materno infantil.

Objetivo Este trabajo tuvo como propósito central identificar los factores asociados con mortalidad neonatal en el municipio Macuspana, estado de Tabasco, México, 2004-2005.

Métodos Se realizó un estudio retrospectivo de casos y controles, los casos fueron 51 fallecidos de 0 hasta 27 días y 153 controles recién nacidos vivos, según lugar de residencia de los padres y fecha de nacimiento.

Resultados Las variables que resultaron asociadas fueron: peso al nacimiento menor de 2500 g OR 12,5 ( IC $\left._{95 \%} 11,62-13,37\right)$, edad gestacional menor de 37

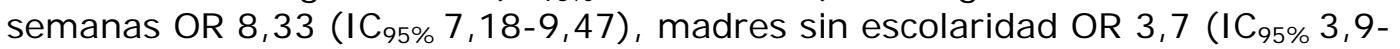
$4,20)$, enfermedad durante el embarazo OR 2,32 (I $\left.\mathrm{C}_{95 \%} 2,28-2,35\right)$, menos de 3 consultas prenatales OR 1,88 (IC $95 \% 1,62-2,13)$.

Conclusiones La mortalidad neonatal es un problema de salud pública en Macuspana, se comprobó que aún existen reservas para la reducción de la mortalidad neonatal si se logra adecuado control del embarazo, diagnóstico 
temprano de las enfermedades, detección oportuna de complicaciones y aseguramiento de la atención hospitalaria a la embarazada y del neonato.

Palabras claves: Mortalidad neonatal, factores asociados, prematurez, bajo peso al nacer.

\section{ABSTRACT}

Introduction Neonatal mortality is a world health problem and at the same time, it is an indicator of the quality of child and maternal care.

Objective This paper was aimed at identifying the factors associated to neonatal mortality in Macuspana municipality located in Tabasco, Mexico in the period 20042005.

Methos A retrospective case-control study was performed. The cases were 51 dead people aged 0 to 27 days and 153 live newborn controls, according to the place of residence of their parents and birth dates.

Results The associated variables were birthweight $<2500 \mathrm{~g}$ OR 12.5 ( Cl95\% 11.62-13.37); gestational age (<37 weeks OR 8.33 (Cl 95\% 7.18-9.47); illiterate mothers OR 3.7 ( $\mathrm{Cl} 95 \%$ 3.9-4.20); disease at pregnancy OR 2.32 ( $\mathrm{Cl}$ 95\% 2.282.35 ), less than 3 prenatal visits to the doctor's, OR 1.88 (IC95\% $1.62-2.13$ ).

Conclusions Neonatal mortality is a public health problem in Macuspana. It was confirmed that there is still possibilities for the reduction of neonatal mortality if adequate control on pregnancy, early diagnosis of diseases, timely detection of complications and assurance of hospital care for the pregnant woman and the neonatum are guaranteed.

Key words: Neonatal mortality, associated factors, prematurity, low birthweight.

\section{NTRODUCCI ÓN}

La Organización Mundial de Salud (OMS) define la mortalidad neonatal como la muerte producida entre el nacimiento hasta los 28 días de vida. Se considera un problema de salud pública y es el indicador básico a considerar para valorar la calidad de la atención en salud del recién nacido en una determinada área geográfica o en un servicio. ${ }^{1}$ En este sentido la OMS recomienda incluir como nacidos vivos a todos aquellos niños que tienen al nacer alguna señal de vida: respiración o palpitaciones del corazón o cordón umbilical, o movimientos efectivos de músculos de contracción voluntaria. ${ }^{2}$

La enfermedad y la muerte del recién nacido están asociadas a diversos factores relacionados con los genes y el medio ambiente, se asocia a la restricción del crecimiento fetal, mujeres con talla menor de $156 \mathrm{~cm}$, embarazos múltiples e hipertensión arterial. En embarazos de peso normal al nacer, el riesgo de muerte neonatal se asocia a la edad de la madre sobre todo en las adolescentes, el hábito de fumar, embarazos múltiples, hipertensión arterial y fundamentalmente las características individuales del neonato. ${ }^{3-6}$ 
En la Cumbre Mundial en favor de la Infancia realizada en 1990 y en la Conferencia Internacional sobre la Población y el Desarrollo de 1994 se incluyeron objetivos en relación con la reducción de los niveles de mortalidad infantil al 2015, proponen una medición intermedia en el año 2000; en este sentido las Naciones Unidas efectuó en el año 2001 una medición que revela avances puesto que la mortalidad infantilse redujo el $11 \%$, pero no alcanzó la meta de reducirse en el $33 \%$ y la mortalidad perinatal en el $30 \% .^{7-10}$

En el 2005 la OMS reportó que cada año mueren cerca de 11 millones de niños, lo que representa unas 30000 muertes por día. De ellas el $40 \%$ ocurre durante el primer mes de vida. ${ }^{11}$

La mortalidad infantil después de los años 70 se convirtió en uno de los principales indicadores de salud para medir el grado de desarrollo alcanzado por un país o una región, a mayor mortalidad infantil menor desarrollo, a menor mortalidad infantil mayor desarrollo, clasificación que llevó a adoptar los términos en países desarrollados y subdesarrollados. ${ }^{12}$

En América Latina se ha avanzado mucho más en la descripción del fenómeno de la mortalidad neonatal que en la explicación de las causas que lo determinan, a pesar de la adversa situación económica se han registrado progresos significativos en los principales indicadores de salud incluyendo reducciones en la mortalidad infantil. Sin embargo, al analizar los promedios regionales o nacionales de esos indicadores se dejan ver las marcadas desigualdades existentes entre los países y al interior de estos. En muchos casos incluso se ha ampliado la brecha entre América Latina y el Caribe y las regiones más desarrolladas del mundo. ${ }^{13,14}$

México con el propósito de acatar los compromisos asumidos en la Cumbre Mundial en favor de la Infancia, en enero de 1991 constituyó la Comisión Nacional de carácter intersectorial e interinstitucional, coordinada por la Secretaría de Salud la que se encargaría de darle seguimiento al cumplimiento de estos objetivos. En junio de ese mismo año se elaboró el documento "México y la Cumbre Mundial en Favor de la Infancia", donde se informó sobre la situación del país al término de 1990, lo que constituyó un diagnóstico y punto de partida para la elaboración del Programa Nacional de Acción. El objetivo principal del programa fue elevar la supervivencia, impulsar el desarrollo y el bienestar de los niños y de las mujeres en edad reproductiva. ${ }^{15}$

El Estado de Tabasco, uno de los más pobres del país, emplazado al sur, donde se ubica el municipio de Macuspana, no ha estado ajeno a estos propósitos y ha asumido acciones concretas que han repercutido de manera muy favorable en el mejoramiento de la salud de la población y en particular de los niños. En este sentido la Secretaria de Salud ha jugado un rol muy importante y positivo, dándole gran prioridad en las inversiones de recursos, creación de instituciones de salud y capacitación de los recursos humanos que trabajan en el sector, lo que ha provocado una importante reducción de la mortalidad infantil, no así en la mortalidad neonatal. El Estado tiene previsto continuar la política de fortalecimiento y mejora continua de la salud de la población, por lo que tiene programado importantes inversiones que requieren ser bien direccionadas para lograr mayor efectividad y eficacia en sus resultados, por otro lado, posee muy pocas investigaciones relacionadas con la salud de la población y sus determinantes y particularmente no hay hechos científicamente fundamentados sobre la mortalidad infantil y sus componentes. Por ello se ha venido realizando un estudio sobre la mortalidad neonatal y sus factores en todo el Estado, del cual forman parte los resultados que sobre el municipio de Macuspana se muestran en este artículo, cuyo 
objetivo es identificar los factores de riesgo asociados a la mortalidad neonatal en Macuspana durante el 2004-2005.

\section{MÉTODOS}

Se realizó un estudio de casos y controles, con un componente descriptivo para identificar las causas de defunción y otro analítico para determinar los factores asociados a la mortalidad neonatal en el periodo 2004-2005 del municipio de Macuspana del Estado de Tabasco.

El universo de estudio lo constituyeron las 55 defunciones neonatales ocurridas en el período de estudio. Se investigaron sólo 51 defunciones neonatales, cuyos padres fueron localizados en el municipio al momento de la investigación. Se excluyeron cuatro fallecidos debido al traslado de su familia fuera del municipio.

Se realizó un estudio de casos y controles para la fase analítica, donde los casos fueron los 51 fallecidos de 0 a 28 días de vida, durante el tiempo de la investigación, residentes del municipio de Macuspana. Se seleccionaron tres controles por cada caso entre los nacidos vivos de 0 a 28 días de vida, que se registraron durante el mismo periodo de nacimiento de los casos, pareados por lugar de residencia de los padres y mes de nacimiento del neonato, para un total de 153 controles.

Para el estudio se utilizaron los datos del Sistema Epidemiológico y Estadístico de Defunciones (SEED) de la Secretaría de Salud del Estado de Tabasco correspondientes al municipio de Macuspana en el periodo 2004-2005, que contiene la información registrada del formato oficial del Certificado de Defunción.

Se diseñó y aplicó un cuestionario a los familiares de los neonatos fallecidos y controles, para identificar los factores asociados. El instrumento fue ajustado previamente en un pilotaje con un grupo de personas que representó el $15 \%$ de los casos de estudio.

Se calcularon como medidas de asociación el Ji cuadrado y para medir la fuerza de asociación se calculó el Odds Ratio (OR), con intervalo de confianza de $95 \%$. Se asumió como diferencias estadísticamente significativas aquellas donde el valor de p fuera inferior a 0,05.

\section{RESULTADOS}

En cuanto a la mortalidad neonatal en el periodo de estudio se registraron 55 defunciones neonatales de un total de 5509 nacidos vivos registrados (NVR) con una tasa de 9,9 $\times 1000 \mathrm{NVR}$, de estas, el 58,2\% ( $n=32)$, correspondió a muertes neonatales tempranas (menores de 7 días de edad), siendo el sexo femenino el mayormente afectado con una razón de 1,6:1. Las principales causas de defunción fueron el síndrome de insuficiencia respiratoria del recién nacido, las infecciones del recién nacido y las malformaciones congénitas, cada una con el 16,4\% tal como aparece en la tabla 1.

Tabla 1. Caracteristicas y causas de muerte neonatal, Macuspana, Tabasco, 20042005 


\begin{tabular}{|c|c|c|c|}
\hline Variable & & No. & $\%$ \\
\hline \multirow[t]{3}{*}{$\begin{array}{l}\text { Edad del } \\
\text { neonato }\end{array}$} & < de 7 días & 32 & 58,2 \\
\hline & 7-27 días & 23 & 41,8 \\
\hline & Subtotal & 55 & \\
\hline \multicolumn{4}{|l|}{ Sexo } \\
\hline & Hombre & 21 & 38,2 \\
\hline & Mujer & 34 & 61,8 \\
\hline & Subtotal & 55 & \\
\hline \multicolumn{4}{|c|}{ Causa de defunción } \\
\hline & $\begin{array}{l}\text { Síndrome de insuficiencia } \\
\text { respiratoria del RN* }\end{array}$ & 9 & 16,4 \\
\hline & Malformaciones congénitas & 9 & 16,4 \\
\hline & Infecciones del RN* & 9 & 16,4 \\
\hline & Síndrome de aspiración neonatal & 8 & 14,5 \\
\hline & Inmadurez/prematurez & 8 & 14,5 \\
\hline & Asfixia del nacimiento severa & 4 & 7,3 \\
\hline & Otras & 8 & 14,5 \\
\hline & Subtotal & 55 & 100,0 \\
\hline
\end{tabular}

* RN: recién nacido.

De un total de 204 casos estudiados e incluidos para el análisis, 51 correspondieron a muertes neonatales $(25,0 \%)$ y 153 a controles $(75,0 \%)$.

En relación con las variables sociodemográficas se observó que más de la mitad de las familias de los neonatos estudiados vivían en área rural, se encontró el mismo comportamiento tanto para casos como para controles; en cuanto a otros datos, se encontró que más del 95,0 \% de las parejas vivían unidas y el 67,0 \% no tenían seguridad social, se destaca, en este último indicador, que para los casos casi fue el doble comparado con los controles (tabla 2).

Tabla 2. Variables sociodemográficas de la mortalidad neonatal, Macuspana, Tabasco, 2004-2005

\begin{tabular}{|l||c|c|c|c|c|c|c|}
\hline Variables & \multicolumn{2}{|c|}{ Casos } & Controles & \multicolumn{2}{c|}{ Total } \\
\hline \hline & & No. & $\%$ & No. & $\%$ & No. & $\%$ \\
\hline Tipo de población & & & & & & \\
\hline \hline & Urbana & 20 & 39,22 & 63 & 41,18 & 83 & 40,69 \\
\hline & Rural & 31 & 60,78 & 90 & 58,82 & 121 & 59,31 \\
\hline & Subtotal & 51 & 100,0 & 153 & 100,0 & 204 & 100,0 \\
\hline \hline
\end{tabular}




\begin{tabular}{|l|l|c|c|c|c|c|c|c|}
\hline & Subtotal & 51 & 100,0 & 153 & 100,0 & 204 & 100,0 \\
\hline Seguridad social & Sí & 25 & 49,02 & 112 & 73,2 & 137 & 67,16 \\
\hline \hline & No & 26 & 50,98 & 41 & 26,8 & 67 & 32,84 \\
\hline \hline & Subtotal & 51 & 100,00 & 153 & 100,0 & 204 & 100,00 \\
\hline
\end{tabular}

En relación con las variables de la madre asociadas a la mortalidad neonatal, su grado de escolaridad y ocupación, se encontró que más del 70,0 \% tenía al menos educación básica y un mínimo porcentaje resultó sin escolaridad; la mayoría de las madres de los neonatos estudiados comunicaron tener como ocupación, las tareas del hogar.

Con repecto al intervalo intergenésico resaltó el hecho de que el 33,0 \% de las madres de los neonatos tuvieron intervalos menores a los 9 meses y que la mayoría de ellas informaron haber tenido 2 o más embarazos previamente. Llamó mucho la atención que más del $90,0 \%$ de las madres de los neonatos recibieron sólo entre 1 y 3 consultas prenatales y la mayoría advirtieron no haber presentado ninguna enfermedad. La mayoría de los partos de los casos y controles se atendieron en una unidad hospitalaria (tabla 3 ).

Tabla 3. Variables de la madre asociadas a la mortalidad neonatal, Macuspana, Tabasco, 2004-2005

\begin{tabular}{|c|c|c|c|c|c|c|c|}
\hline \multirow[t]{2}{*}{ Variables } & & \multicolumn{2}{|c|}{ Casos } & \multicolumn{2}{|c|}{ Controles } & \multicolumn{2}{|c|}{ Total } \\
\hline & & No. & $\%$ & No. & $\%$ & No. & $\%$ \\
\hline \multicolumn{8}{|c|}{ Escolaridad de la madre } \\
\hline & $\begin{array}{l}\text { Sin } \\
\text { escolaridad }\end{array}$ & 3 & 5,88 & 3 & 1,96 & 6 & 2,94 \\
\hline & $\begin{array}{l}\text { Educación } \\
\text { básica }\end{array}$ & 41 & 80,39 & 105 & 68,63 & 146 & 71,57 \\
\hline \multirow[t]{2}{*}{$\cdots$} & $\begin{array}{l}\text { Educación } \\
\text { superior }\end{array}$ & 7 & 13,73 & 45 & 29,41 & 52 & 25,49 \\
\hline & Subtotal & 51 & 100,0 & 153 & 100,0 & 204 & 100,0 \\
\hline \multicolumn{8}{|c|}{ Ocupación de la madre } \\
\hline & Campo & 0 & 0 & 2 & 1,31 & 2 & 0,98 \\
\hline & Empleada & 5 & 9,8 & 19 & 12,42 & 24 & 11,76 \\
\hline & $\begin{array}{l}\text { Trabaja por } \\
\text { su cuenta }\end{array}$ & 0 & 0 & 8 & 5,23 & 8 & 3,92 \\
\hline & Estudia & 0 & 0 & 3 & 1,96 & 3 & 1,47 \\
\hline & Hogar & 46 & 90,2 & 121 & 79,08 & 167 & 81,86 \\
\hline & Subtotal & 51 & 100,0 & 153 & 100,0 & 204 & 100,0 \\
\hline \multicolumn{8}{|l|}{$\begin{array}{l}\text { Intervalo } \\
\text { intergenésico }\end{array}$} \\
\hline & $<9$ meses & 16 & 31,4 & 52 & 34 & 68 & 33,3 \\
\hline
\end{tabular}




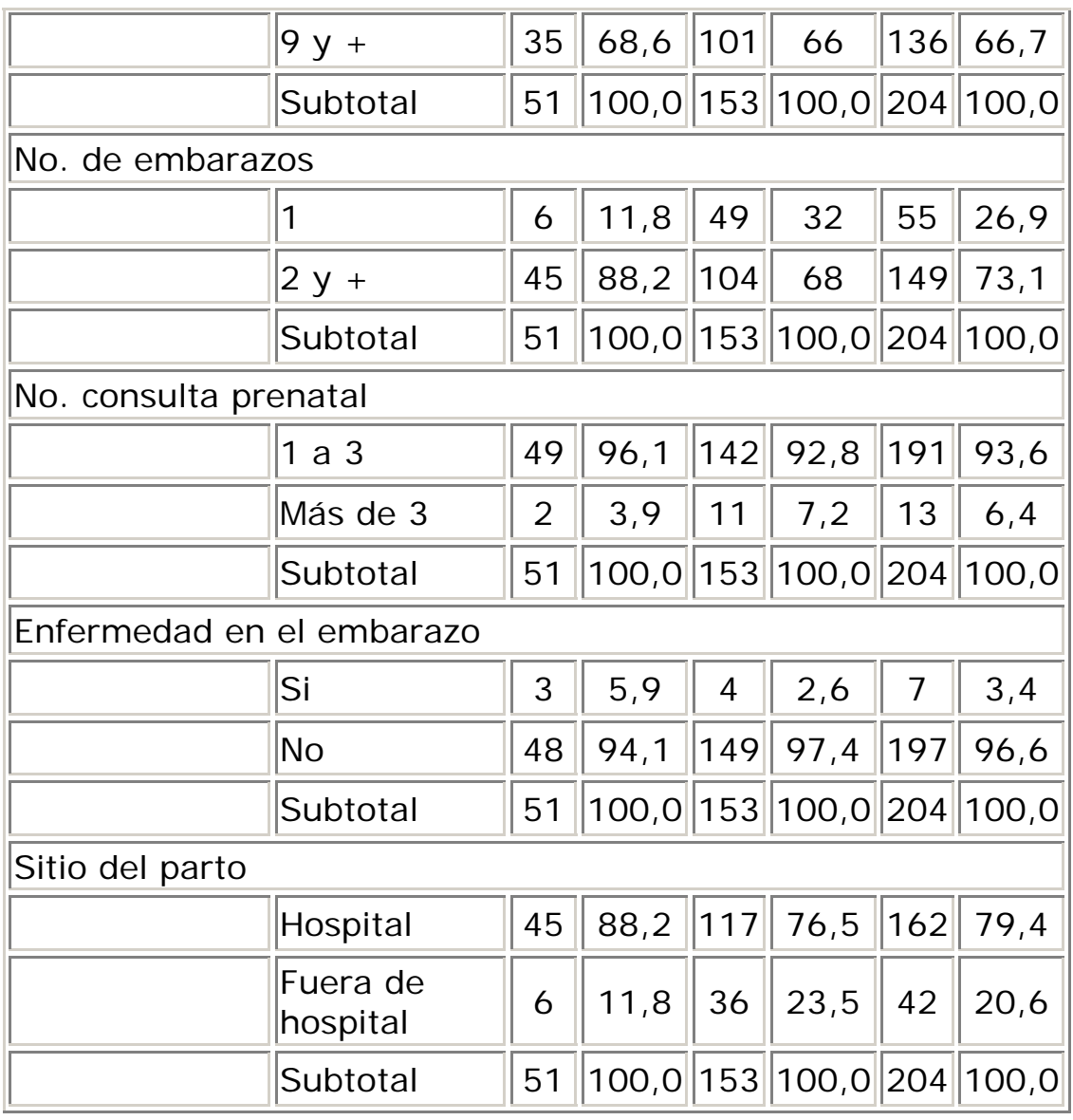

En la tabla 4 se muestra que del total de defuncines y en relación con el sexo y la ocupación de la madre, la distribución fue muy similar, sin embargo, se observa que en los casos el mayor número de muertes ocurrió en el sexo femenino.

En relación con la edad del producto en semanas de gestación, se aprecia que del total, la mayoría de los neonatos tuvieron una edad gestacional igual o mayor a 37 semanas, cabe resaltar que para los controles la distribución es poco mas del doble a la observada para los casos.

En cuanto al peso en gramos al nacimiento, los resultados mostraron que el 43,1\% de los recién nacidos fallecidos tenían bajo peso, menor de $2500 \mathrm{~g}$; para los controles esta situación sólo se presentó en el 5, 9 \%.

Tabla 4. Variables del neonato asociadas a la mortalidad neonatal. Macuspana, Tabasco, 2004-2005

\begin{tabular}{|l|c|c|c||c|c||c|c||}
\hline Variables & & \multicolumn{2}{|c|}{ Casos } & Controles & \multicolumn{2}{c|}{ Total } \\
\hline \hline & & No. & $\%$ & No. & $\%$ & No. & $\%$ \\
\hline Sexo & Hombre & 19 & 37,3 & 81 & 52,9 & 100 & 49 \\
\hline \hline & Mujer & 32 & 62,7 & 72 & 47,1 & 104 & 51 \\
\hline \hline & Subtotal & 51 & 100,0 & 153 & 100,0 & 204 & 100,0 \\
\hline Edad gestacional del producto & & & & \\
\hline & $<37$ & 30 & 58,8 & 22 & 14,4 & 52 & 25 \\
\hline & $37 \mathrm{y}+$ & 21 & 41,2 & 131 & 85,6 & 152 & 75 \\
\hline
\end{tabular}




\begin{tabular}{|l|c|c|c|c|c|c|c|c|}
\hline & Subtotal & 51 & 100,0 & 153 & 100,0 & 204 & 100,0 \\
\hline Peso del producto en gramos \\
\hline \hline & $<2500$ & 22 & 43,1 & 9 & 5,9 & 31 & 15 \\
\hline \hline & $2500 \mathrm{y}+$ & 29 & 56,9 & 144 & 94,1 & 173 & 85 \\
\hline & Subtotal & 51 & 100,0 & 153 & 100,0 & 204 & 100,0 \\
\hline \hline
\end{tabular}

En la tabla 5 se presentan las variables que resultaron asociadas con las muertes de neonatos en el periodo de estudio: la que resultó con mayor asociación fue el peso del producto menor a $2500 \mathrm{~g}$, con un OR de 12,5 (IC $95 \% 11,62-13,37)$; la segunda variable con mayor asociación fue la edad del producto menor a 37 semanas, y un OR 8,33 ( I $\left._{95 \%} 7,18-9,47\right)$; la madre sin escolaridad presentó un OR $3,70\left(I_{95 \%} 3,19-4,20\right)$; la presencia de enfermedad de la madre durante el embarazo, OR de 2,32 (IC $95 \% 2,28-2,35)$; finalmente menos de 3 consultas prenatales un OR de $1,88\left(\mathrm{IC}_{95 \%} 1,62-2,13\right)$.

Tabla 5. Variables asociadas a la mortalidad neonatal Macuspana, Tabasco, 20042005

\begin{tabular}{|c|c|c|c|c|c|c|c|c|}
\hline $\begin{array}{c}\text { Peso del producto } \\
\text { en gramos }\end{array}$ & \multicolumn{2}{|c|}{ Casos } & \multicolumn{2}{|c|}{ Controles } & & & \multicolumn{1}{|c|}{} \\
\hline & No. & $\%$ & No. & $\%$ & $\mathrm{Ji}^{2}$ & OR & $\mathrm{IC}_{95 \%}$ \\
\hline$<2500$ & 22 & 43,14 & 9 & 5,88 & 41,2 & 12,5 & $\begin{array}{c}11,62- \\
13,37\end{array}$ \\
\hline 2500 y + & 29 & 56,86 & 144 & 94,12 & - & 1 & - \\
\hline \hline Subtotal & 51 & 100,0 & 153 & 100,0 & & & \\
\hline
\end{tabular}

Edad gestacional del producto

\begin{tabular}{|l|l|l|l|l|l|l|l|c||}
\hline$<37$ & 30 & 58,82 & 22 & 14,38 & 39,78 & 8,33 & $\begin{array}{c}7,18- \\
9,47\end{array}$ \\
\hline $37 \mathrm{y}+$ & 21 & 41,18 & 131 & 85,62 & - & 1 & - \\
\hline Subtotal & 51 & 100,0 & 153 & 100,0 & & & \\
\hline
\end{tabular}

Escolaridad de la madre

\begin{tabular}{|l|c|c|c|c|c|c|c|}
\hline Sin escolaridad & 3 & 5,88 & 3 & 1,96 & 2,06 & 3,7 & $\begin{array}{c}3,19- \\
4,20\end{array}$ \\
\hline Educación básica & 41 & 80,39 & 105 & 8,63 & 2,6 & 0,53 & $\begin{array}{c}0,24- \\
1,15\end{array}$ \\
\hline $\begin{array}{l}\text { Educación } \\
\text { superior }\end{array}$ & 7 & 13,73 & 45 & 29,41 & - & 1 & - \\
\hline \hline Subtotal & 51 & 100,0 & 153 & 100,0 & & & \\
\hline
\end{tabular}

Enfermedad en el embarazo

\begin{tabular}{|l|l|l|l|l|l|l|c|}
\hline Sí & 3 & 5,88 & 4 & 2,61 & 1,23 & 2,32 & $\begin{array}{c}2,28- \\
2,35\end{array}$ \\
\hline No & 48 & 94,12 & 149 & 97,39 & - & 1 & - \\
\hline \hline & 51 & 100,0 & 153 & 100,0 & & & \\
\hline
\end{tabular}

No. de consulta prenatal

\begin{tabular}{|l|l|l|l|l|l|l|l|l|}
\hline 1 a 3 & 49 & 96,08 & 142 & 92,81 & 0,68 & 1,88 & $\begin{array}{c}1,62- \\
2,13\end{array}$ \\
\hline
\end{tabular}




\begin{tabular}{|l||c|c|c|c|c|c|c||}
\hline Más de 3 & 2 & 3,92 & 11 & 7,19 & - & 1 & - \\
\hline \hline Subtotal & 51 & 100,0 & 153 & 100,0 & & & \\
\hline
\end{tabular}

\section{DISCUSIÓN}

El presente estudio mostró que más del $50 \%$ de las muertes estuvieron asociadas al nacimiento pretérmino, y solamente el $43 \%$ con bajo peso al nacer, estos resultados son similares con lo encontrado en un hospital de Colombia en el $2001^{16}$ que se basó también en un diseño de casos y controles que demostró que la muerte neonatal está asociada a factores patogénicos que intervienen en forma simultánea.

Existen numerosos informes en la literatura, donde el peso al nacer y la edad gestacional se asocian significativamente con la mortalidad neonatal, tal como el que se realizó en Lima Perú ${ }^{3}$ en el 2003 donde se comprobó que la muerte fetal y neonatal ocurren por factores patogénicos de origen multifactorial y la mayor parte de estas ocurren por factores que intervienen tempranamente, de manera que la salud física, mental y social de la madre está estrechamente ligada al bienestar del hijo antes de nacer y a la salud del hijo después del nacimiento. En sus resultados encuentran asociación de la mortalidad neonatal con la primera gestación, el bajo peso al nacer y el antecedente de tener más de tres partos. En el presente estudio se encontró asociación con el bajo peso al nacer pero no con las otras variables.

En relación con la edad materna, en la literatura está sustentada su asociación con el bajo peso al nacer, tanto que varios autores proponen que esta se estandarice como predictor para la mortalidad neonatal en edades menores de 18 años y mayores de $35 .{ }^{4}$ Otros autores ${ }^{17}$ han encontrado un aumento en la incidencia de mortalidad neonatal entre las adolescentes embarazadas quienes presentan mayor riesgo de abortos espontáneos, productos con bajo peso al nacer y antecedentes de muertes neonatales previas, pero no refieren la edad materna como una de sus causas. Sin embargo, la edad materna en el presente estudio no mostró diferencia estadísticamente significativa.

Dentro de las características sociodemográficas que se encontraron en el presente estudio como factores asociados a la mortalidad neonatal fueron: madres de neonatos sin escolaridad y madres con ocupación dedicada al hogar. Datos que están de acuerdo con otros hallazgos de la literatura que sustentan que las condiciones sociales, culturales, demográficas y económicas están asociadas para que se presente este evento. ${ }^{18}$

En el presente estudio el rango de consultas prenatales fue el mas bajo, entre 1 y 3 consultas otorgadas a la madres de los neonatos fallecidos; algunas investigaciones, enfatizan la importancia no sólo del número de consultas sino la oportunidad y efectividad del control prenatal y señalan el impacto en complicaciones del parto y bajo peso al nacimiento. ${ }^{19}$

Finlamente se puede concluir que la mortalidad neonatal es un problema de salud pública en el municipio de Macuspana del Estado de Tabasco dado los hallazgos encontrados en la presente investigación sobre los factores asociados a la muertes neonatales como son: el bajo peso al nacer, la alta tasa de prematurez y otras características de la madre, baja o nula escolaridad y el bajo número de consultas prenatales. 
Existen posibilidades para la reducción de la mortalidad neonatal dado que muchas de las causas de muerte son prevenibles, entre ellas, las infecciones en el recién nacido, las relacionadas con la atención del parto, y aún las malformaciones congénitas, pero para esto sería necesario involucrar al personal de las unidades de la atención primaria del sistema estatal de salud, para que la mujer lleve un adecuado control del embarazo que permita un diagnóstico temprano de las enfermedades, una detección oportuna de las complicaciones del recién nacido y asegurar una atención en medio hospitalario.

Algunas recomendaciones útiles serían:

- Implementar de forma ineludible, estrategias para asegurar la cobertura de la atención prenatal en las mujeres del municipio desde fases tempranas del embarazo e incrementar el número de consultas conforme a los trimestres de evolución del embarazo.

- Reforzar las acciones de capacitación sistemática del personal de la salud del primer nivel de atención relacionadas con la identificación temprana de los factores de riesgo tanto en la madre como en el producto a fin de contribuir a la reducción de la mortalidad neonatal.

- Continuar las investigaciones que incorporen la mortalidad perinatal, a fin de estudiar las características de los niños no nacidos. Lo anterior es importante partiendo de los resultados obtenidos en este estudio donde se logró identificar los factores asociados a la mortalidad neonatal.

\section{REFERENCI AS BI BLI OGRÁFICAS}

1. Tena C, Ahued J R. Recomendaciones generales para mejorar la calidad de la atención materno infantil. Ginecol Obstet Méx. 2003; 71:409-20.

2. World Health Organization. Estimates: Saving newborn lives. State of world's newborns. Washington: WHO;2001. [Save the Children Federation-US].

3. Svitone EC, Garfield R, Vasconcelos MI, Araujo V. Primary health care from the Northeast of Brazil: the agents de saúde program. Pan Am Public Health.

2000; 7: 293-301.

4. Huiza L, Pacora P, Máximo Ayala M, Buzzio Y. La muerte fetal y la muerte neonatal tienen origen multifactorial Ann Fac. 2003;64: 1-7. [Lima Perú].

5. Simon L. Morbilidad materna en la adolescente embarazada. Rev Ginecol Obstet Méx. 2002; 70:270-4.

6. Word Health Organization. Department of Reproductive Maternal and New born Health and Research (RHR). Guia práctica. 2003;112:45-7.

7. Organización de Naciones Unidas. Informe sobre la Conferencia Internacional de Población y Desarrollo, El Cairo, Egipto: ONU; 1994.

8. Benguigui Y. Acciones de salud maternoinfantil a nivel local: según las metas de la Cumbre Mundial en favor de la infancia. México, D.F. Cumbre

Mundial; 1999(12): 4-12. 
9. CRIN y UNICEF. Informe de seguimiento de la década a la Cumbre Mundial en favor de la infancia. New York: ONU; 2002. [Sesión Especial de las Naciones Unidas].

10. Conferencia Sanitaria Panamericana. 54.a Sesión del Comité General. Washington, D.C.: Comité General; 2002.

11. Organización Panamericana de la Salud. Día Mundial de la Salud: Informe Anual del Director Washington, D.C.: OPS; 2005.

12. UNDP. Actores internacionales en la Educación Básica de Adultos. Educación de Adultos y Desarrollo (Suplemento); 2003.

13. Pierdant M, Ávila C. Análisis de la mortalidad en el Hospital Infantil de México Federico Gómez de 1943 a 1999: un reflejo de la transición epidemiológica. Bol Med Hosp. 2000; 58(9):599-614.

14. Alazraqui M. Mortalidad Infantil. Un indicador para la Gestión Local. Análisis de la Mortalidad Infantil de la Provincia de Buenos Aires en 2001. OPS Publicación Científica $N^{\circ} 51$. Buenos Aires: OPS; 2001.

15. Comisión Nacional de Acción en Favor de la Infancia. Informe Nacional sobre el Seguimiento de la Cumbre Mundial de la Infancia, México,DF: Comisión; 2000.

16. Delgado $M$, Muñoz A, Orejuela L, Sierra H. Algunos factores de riesgo para mortalidad neonatal en un hospital de III nivel, Popayán. Colomb Med. 2003; 34: 179-85.

17. Cáceres Manrique FM, Meza Durán E, Arias Barrera C. Factores de riesgo asociados a la mortalidad neonatal. Hospital Universitario Ramón González. Valencia, España. Rev MEDUNAB. 2001;4(11): 107-14.

18. Recaro J, Torrents A. Algunos apuntes sobre los determinantes sociodemográficos de la mortalidad infantil en Cataluña (s. XVIII-XX). Menorca, España: Asociación de Demografía Histórica (ADEH), Institut Menorquí d'Estudis Maó; 2003. [I Encuentro de Demografía Histórica de la Europa Meridional].

19. Alfaro. Oportunidad y continuidad de la atención prenatal en embarazos de riesgo bajo y frecuencia de cesáreas. Rev Med IMSS. 2002;40 (5):415-9.

Recibido: 11 de julio de 2007.

Aprobado: 24 de septiembre de 2008.

Carlos Mario de la Cruz Gallardo. Secretaría de Salud del Gobierno del Estado de Tabasco. México. 
